\title{
Domesticación, bienestar y relación entre el perro y los seres humanos
}

\author{
Koscinczuk, P. \\ Cátedra de Patología Médica, Facultad de Ciencias Veterinarias, Universidad Nacional del Nordeste, \\ Sargento Cabral 2139, Corrientes (3400), Argentina. Tel. 03794-425753. \\ E-mail: pkoscinczuk@hotmail.com
}

\begin{abstract}
Resumen
Koscinczuk, P.: Domesticación, bienestar y relación entre el perro y los seres humanos. Rev. Vet. 28: 1, 78-87, 2017. La especie canina es la que mejor se ha adaptado a vivir junto al hombre. Prueba de ello es que existen poblaciones de perros distribuidas en todos los continentes, a excepción de la Antártida. A través de un largo proceso de domesticación, los perros han modificado su apariencia física y su comportamiento. Las razas más antiguas, descendientes de los lobos, se originaron en África y Asia. No obstante, la gran explosión en el aumento de la población de perros tuvo lugar en Europa a partir del año 1800. Mediante la cría selectiva basada en el fenotipo y dirigida por el hombre, han surgido decenas de razas, en las que los individuos son reconocidos y clasificados según rasgos físicos característicos. Pueden encontrarse situaciones complejas, que van desde aquéllas que afectan al estado de salud (prognatismos, displasias, acondroplasia), hasta aquéllas que involucran a la sociabilidad con el ser humano y dan lugar a emociones negativas como miedo o ansiedad. El hecho de que el perro sea considerado un miembro más de la familia no significa que deba ser forzado a vivir según las necesidades de la familia humana. Los perros tienen necesidades conductuales y una comunicación propia. El ritual de la comida, el lugar donde descansan y desarrollan sus actividades, y otros hábitos influyen fuertemente en su bienestar. Es un gran avance que los animales sean reconocidos como individuos con derechos, pero no es suficiente. Es necesario enseñar conceptos básicos sobre las necesidades de los animales, para evitar que sufran innecesariamente: concientizar acerca del no abandono, del control de la reproducción y de la prevención de las patologías hereditarias, entre otros. Solo teniendo en cuenta los puntos mencionados, las posiciones éticas serán motivo de discusión y la jurisprudencia podrá obrar de manera adecuada.
\end{abstract}

Palabras clave: canino, necesidades básicas, alimentación, socialización.

\begin{abstract}
Koscinczuk, P.: Domestication, comfort, and relationship between the dog and human beings. Rev. Vet. 28: 1, 78-87, 2017. The dog is one of the species best adapted for living with humans that inhabits all the continents except Antarctica. After a long process of domestication, dogs have modified not only physical appearance but also their behavior. The most antique breeds descend from wolves were originated in Africa and Asia. However, the biggest increase in dogs' population had taken place in Europe since the 1800s. Through selective breeding, managed by men and based upon the phenotype, hundreds of breeds had emerged, and each one is recognized and classified according to specific physical features. Other classification takes into account dogs' dependence on humans in order to obtain food and shelter. When it comes to evaluate domestic dog's welfare such diversity of classifications should be considered. Some complex situations regarding health (prognathous, dysplasia, acondrodysplasia) as well as those associated with negative emotions -such as fear and anxiety- that affect sociability with humans may arise due to undesirable fixation of some genes in particular breeds. In some cases dogs are considered as members of the family but this does not mean that they must live according to human needs. Dogs have behavior needs and their own way of communication. Feeding rituals, resting places, playing areas, and others practices have an important impact on their wellness. Animals must be protected and the implementation of animal rights is a huge progress, but it is still not enough. Education is also essential. It is necessary to teach basic concepts about animal needs to avoid unnecessary suffering: pet abandonment, control of reproduction and inheritable pathologies, among others. Only after
\end{abstract}


these conditions are met it will be possible to discuss from different points of view and perhaps jurisprudence may properly work.

Key words: canine, basic needs, feeding, socialization.

\section{PRÓLOGO}

El hombre no puede eludir las responsabilidades morales que tiene sobre el perro. Esta especie no solo ha modificado su comportamiento sino también su estructura física para poder vivir en el ambiente social humano. Peter Singer, filósofo contemporáneo australiano, destaca el hecho de que un periódico de la relevancia del New York Times se refiera al perro utilizando los pronombres él o ella en lugar del neutro "lo" (del inglés she or he en lugar de it) ${ }^{55}$.

En muchas oportunidades, el perro es considerado como un integrante más de la familia. Esta compatibilidad entre el perro y el hombre no es nueva. Al estudiar los rituales de entierro, los antropólogos destacan que, en ocasiones, las personas responden a la muerte de su perro de la misma manera que responde a la muerte de un miembro de la familia. En excavaciones realizadas en Ashkelon, Israel, se encontraron restos de esqueletos de alrededor de 1000 perros enterrados deliberadamente 2.000 años atrás. Estos perros seguramente murieron por causas diversas pero, seguramente, no por maltrato ${ }^{57,62}$.

Esta relación social especial cada vez se ve más fortalecida con la creación de numerosos y modernos cementerios para perros en diferentes partes del mundo ${ }^{38}$. Más allá de estos rituales que envuelven la relación humano animal, el proceso de domesticación es complejo y abarca cambios no sólo físicos sino también de comportamiento, que son heredados de generación en generación ${ }^{48}$. Conocer el proceso de domesticación nos permite entender un poco más la relación del perro actual con el ser humano y entender las necesidades de esta especie para mejorar su bienestar.

\section{DEL PROCESO DE DOMESTICACIÓN}

Se entiende por domesticación a una forma única de mutualismo que se desarrolla entre una población humana y una determinada cantidad de plantas o animales con fuertes ventajas selectivas entre ambas partes. Es cierto que el primer requerimiento para un domesticación animal exitosa es que el hombre, el domesticador, tuviera una necesidad o deseo reconocido que solo habría sido satisfecho mediante el control, protección y cría de cierta población de animales ${ }^{11}$.

En este contexto, el término domesticación se utiliza para designar un proceso histórico por el cual algunos animales o plantas salvajes fueron transformados por el hombre ${ }^{34}$, y donde es el hombre quien los ha domesticado sometiéndolos a cautiverio, criándolos y seleccionándolos por su mansedumbre, incrementado una dependencia mutua ${ }^{64}$. Sin embargo, esto no expli- ca por qué sólo algunos animales evolucionaron a la categoría "perro doméstico", mientras que sus ancestros -el lobo o el chacal- siguen siendo salvajes.

De manera evolutiva, una población de animales se adapta al hombre y a su ambiente de cautiverio ${ }^{6}$. Como todo proceso, no tiene comienzo ni fin, sino que es algo que está en continuo cambio. En este proceso muchas especies, no solo el perro, continúan modificando tanto su aspecto físico como su conducta. La adaptación al ambiente de cautiverio que significa el entorno social humano, apunta a cambios genéticos que ocurren de generación en generación como respuesta a la estimulación ambiental y su experiencia de vivir junto al hombre.

Entender el proceso de domesticación no es fácil porque en realidad estamos hablando de cambios que sucedieron a lo largo del tiempo en las dos especies, tanto en el perro como en el hombre. El hombre evolucionó en lo cultural y tecnológico y los animales evolucionaron acompañando al ser humano. En medio de estas consideraciones hay factores abióticos como el clima y los cambios en la superficie terrestre que, a su vez, afectaron fuertemente a otros factores bióticos como la comida, la presencia de animales competidores y otros predadores. El mundo en el cual comenzó lo que se conoce como período de pre-domesticación era muy distinto al mundo actual ${ }^{63}$.

Se considera que un factor clave que favoreció la domesticación fue el comportamiento parental. Se reconoce que las especies precoces cuyos jóvenes pudieron ser separados tempranamente de sus padres, fueron los más fáciles de domesticar. En cambio las especies altriciales (desvalidas, con un periodo de cuidado parental más prolongado), podrían ser especies donde la domesticación fue más difícil. Sorprendentemente, este no es el caso del perro ni del gato, ya que sus crías nacen sin poder controlar la temperatura corporal, comienzan a ver y oír después de los catorce días de vida ${ }^{31}$ y sin embargo, son dos de las especies domesticas más populares ${ }^{18}$.

\section{COMIENZOS DE LA DOMESTICACIÓN}

Con el avance de la genética molecular hay más evidencia de que el perro desciende del lobo, sugiriéndose que tal vez la población de lobos primitivos haya comenzado el proceso de domesticación entre 50.000 y 100.000 años atrás. Si el origen de la humanidad está en África, es probable que la pre-domesticación del perro también tenga su origen en dicho continente. Desde allí, perros y hombres habrían llegado al Asia para luego expandirse a Europa. Arqueológicamente no es fácil definir el lugar o el momento exacto ${ }^{61}$. 
Los estudios realizados en las excavaciones no son concluyentes, las modificaciones físicas como la reducción del tamaño corporal, la estructura del cráneo y hocico, los dientes más pequeños y amontonados, son hallazgos tardíos ${ }^{34}$. Sin embargo, en los primeros estudios que reconocieron la evolución del perro doméstico, fueron estas modificaciones anatómicas las que se tuvieron en cuenta para diferenciar al perro del lobo, de allí la falta de precisión cronológica sobre el comienzo de la domesticación.

La teoría de evolución del perro es interesante. Hay pruebas que el perro doméstico (Canis lupus familiaris) proviene del lobo (Canis lupus), pero es muy difícil concebir la idea de que el hombre quisiera domesticar al lobo para obtener un beneficio capturándolo, amansándolo o criándolo selectivamente de la misma manera que lo hizo con otros animales. Es probable que los ancestros del lobo se aproximaran al hombre en el periodo mesolítico, viviendo de sus restos de comida y excremento. Estos animales, bien alimentados, se reprodujeron con éxito, a pesar del riesgo que significaba estar cerca de los seres humanos. Estas poblaciones de lobos comenzaron naturalmente a seleccionarse por su mansedumbre sin que necesariamente participen los seres humanos, por lo menos al comienzo del proceso, y se fueron transformando paulatinamente en poblaciones de perros ${ }^{12}$.

El número de perros que conformaba el grupo dependía de la disponibilidad de alimento y generalmente no eran más de tres. Las poblaciones primitivas no fueron conscientes del proceso de amansamiento de estos perros y tampoco los admitían dentro de sus hogares, no obstante ellos toleraron su presencia e inadvertidamente los proveyeron de un nicho adecuado para convivir. Si esta teoría es correcta, el hombre modificó su estilo de vida a favor del perro. Por esto, se considera más plausible considerar que el perro inició su propia domesticación y que el ser humano fue su domesticador muy tarde en este proceso. En todas las especies la intensificación de la relación con los seres humanos comenzó con una antropofilia, continuó con el comensalismo y sólo hacia el final del proceso el hombre ejerció un control de los animales salvajes, proceso que primero se intensificó en la etapa del cautiverio en fase extensiva y luego en la fase de cría intensiva para pasar a ser considerados como mascotas ${ }^{60}$.

En términos evolutivos, el número de animales de una especie se utiliza para describir su adaptación. Evolutivamente, la especie canina es una de las más exitosas. Los perros no solo existen en gran número, sino también son una de las pocas especies de mamíferos que se han distribuido por todo los continentes, excepto la Antártida ${ }^{26}$. Pero desde el punto de vista del perro como individuo, este éxito es cuestionable. El aumento explosivo del número de perros acompañado por el crecimiento de las razas, ha dado lugar a cruzamientos endogámicos con la aparición de numerosos enfermedades ${ }^{21}$.
Mientras que en otros animales los cruzamientos genéticos se hicieron para el beneficio de los seres humanos (más carne, más leche, más lana), en el perro fueron dirigidos a lograr características fenotípicas particulares en pos de algún beneficio para la especie canina. Para mencionar un ejemplo, en el Lhasa apso, raza de origen tibetano, la selección basada en la longitud del pelo tenía por función beneficiar a los individuos protegiéndolos del frío y de las radiaciones solares. Los perros no beneficiaron al hombre primitivo. A lo mejor, favorecieron algunas estrategias de caza, pero en general, las documentaciones sólo describen mutua compañía ${ }^{34}$.

\section{EL MIEDO Y LA TOLERANCIA AL CONTACTO HUMANO}

En un libro que detalla el concepto de "bienestar" se define al perro como un animal cuyo comportamiento es básicamente similar al de su progenitor el lobo, pero con cambios durante la domesticación. Desde el punto de vista conductual, el perro se define como un cazador furtivo, promiscuo, carroñero, social, territorial, con propensión al ladrido y que usa la orina y la materia fecal para comunicarse y sus dientes para defenderse o agredir ${ }^{56}$.

Otros autores identifican seis clases de perros de acuerdo a su relación con el ser humano: salvaje (salvaje por años, como lo es el "dingo"), asilvestrado (salvaje por generaciones), vagabundo sin dueño (abandonado y nacido de hembras vagabundas), vagabundo del vecindario (propiedad del vecindario, todos le dan de comer, pero nadie se responsabiliza por él), vagabundo con familia (propiedad de un individuo que no lo tiene encerrado en su casa) y restringido (propiedad de un individuo, con restricción de las salidas). De esta manera, se puede argumentar que las diferentes clases de perros están definidas por el nivel de restricción impuesto por el hombre (su propietario), por la disponibilidad de recursos (refugio y comida), y por la presión de depredación hacia el ser humano y otras especies ${ }^{32}$.

Sin embargo, esta clasificación no tiene en cuenta la socialización inter-específica (socialización humano-animal). Teniendo en cuenta la ontogenia, se ha propuesto una clasificación ligeramente diferente, considerando las respuestas de miedo y tolerancia que pueden afectar a la relación humano animal. Así, existen perros "restringidos" (condicionados, coartados) y perros familiares, que tienen un propietario del cual dependen, pero pueden quedar libres para deambular. La tercera categoría, los perros vagabundos, constituyen un grupo heterogéneo que comprenden: a) individuos que pese a estar abandonados todavía tienen contacto social con el hombre; b) perros con diferente grado de miedo/tolerancia hacia los seres humanos, atraídos porque les proporcionan comida y refugio ("perros urbanos") ${ }^{5}$. La cuarta categoría son los perros salvajes, que incluyen a los individuos libres, que perviven en estado indómito, sin comida ni protección otorgada por seres humanos y sin ninguna evidencia de socialización con ellos ${ }^{64}$. 
En los países del tercer mundo con grandes extensiones de tierra, donde grupos de perros salvajes viven lejos del control humano, la falta total de contacto social humano desde el nacimiento es un hecho poco frecuente que se puede dar en casos excepcionales en áreas rurales, montes, selva o zonas montañosas ${ }^{15}$. Estos perros practican el vagabundeo, comen restos de comidas e intercambian contacto sexual con perros socializados. Por lo general viven en grupos y causan accidentes atacando a los animales de granja (aves, ovejas, incluso vacas) generando conflictos con otras especies tanto de granja como silvestre ${ }^{22}$.

En tales grupos, aunque las hembras se alimentan de deshechos, generalmente gozan de una salud más o menos buena y entran en celo dos veces al año con una tasa de reproducción alta, generando serios conflictos. Hay reportes que mencionan la disminución del número de individuos de especies protegidas, al ser afectadas por perros salvajes. En los andes tropicales se reconocen unas 13 especies protegidas, que están siendo amenazadas por perros salvajes ${ }^{22}$.

La categorización sistemática y ecológica de los perros vagabundos está sujeta a una considerable discusión, sin embargo los investigadores no encuentran diferencias genotípicas o fenotípicas entre ambos grupos. Por este motivo, debería considerarse que el proceso por el cual un perro doméstico se vuelve salvaje, no es necesariamente la reversa del proceso de domesticación, sino la falta de socialización temprana con la especie humana ${ }^{16}$.

\section{APARICIÓN DE LAS RAZAS}

Una de las maneras de clasificar a los perros se basa en la carga genética. Los genetistas investigan marcas del proceso de domesticación en el genoma de las especies domesticadas, en busca de evidencia del comportamiento humano asociado no solo a cambios genéticos sino también morfológicos ${ }^{65}$. En este aspecto y teniendo en cuenta sus orígenes, se pueden observar dos grupos. Por un lado las razas más antiguas originarias de África/Asia y las más modernas, que proliferaron en Europa a partir del siglo XIX ${ }^{47}$.

En el primer caso, es probable que los cruzamientos hayan sido por selección natural. En el segundo, se destacan los cambios producidos por la presión de selección que, a partir del 1800, los humanos ejercieron sobre la especie canina basados en el fenotipo. En este caso se observa que diferentes razas son genéticamente distintas, y los individuos pueden ser fácilmente asignados a razas sobre la base de su genotipo. "A primera vista, sorprende la agrupación de un clúster genético simple que incluye a las razas de África central (basenji), del medio este (salami y afgán), del Tíbet (terrier tibetano e lhasa apso), de China (chow chow, pekinés, sharpei y shi tzu), de Japón (akita y shiba inu) y del ártico (alaskan malamute, siberian husky y samoyedo)". Sin embargo, al considerar la carga genética del resto de las razas, parece ser el resultado de una propagación más reciente desde el stock europeo. Esta radiación probablemente refleja la proliferación de las distintas razas desde la codificación genotípica y variaciones después de la introducción del concepto de cría y creación de clubes de cría en Europa en $1800{ }^{47}$.

Por presión de selección dirigida por el hombre sobre algunas características fenotípicas se han consolidado las diferentes razas. La forma tradicional y más difundida de categorizar las razas ha sido mediante la promoción de organizaciones de clubes internacionales en los cuales las razas son organizadas en grupos de acuerdo a sus habilidades. Si bien existen varias organizaciones, son dos las que asumen más fuerza de representación: American Kennel Club (AKC) con 180 razas reconocidas integrando 7 grupos y la Federation Cynologique Internationale (FCI) con 365 razas, integrando 10 grupos. Lamentablemente el inbreeding, cruzamiento entre líneas genéticas emparentadas, no solo fijó belleza y habilidades conductuales sino también enfermedades ${ }^{33}$.

Algunas afecciones hereditarias están tan difundidas que es dable hablar de problemas de la raza, en este caso un ejemplo es la displasia de cadera en el ovejero alemán, la sordera del dálmata, o la amiloidosis del sharpei. En otras es posible identificar una línea de sangre que transmite el problema, como la displasia de codo del labrador y la demodexia del bóxer. Pero ¿qué sucede con sus mestizos? En este caso son los veterinarios los que deberían cuidar y manejar las prácticas de manera que estos animales no se reproduzcan, para disminuir el pool genético afectado, preservando la diversidad del pool genético para evitar que las patologías se transmitan de generación en generación ${ }^{49}$.

\section{EL BIENESTAR DEL PERRO DOMÉSTICO}

El proceso de domesticación implica una transición desde lo natural al cautiverio y donde lo natural y el cautiverio son dos extremos de un proceso continuo. El proceso de domesticación resulta en la pérdida de algunos comportamientos del repertorio de la especie. En casi todos los casos, la diferencia es cuantitativa ${ }^{49}$. Las capacidades del perro primitivo fueron transformadas específicamente por el desafío que significó vivir con el hombre, desarrollando habilidades sociales características de la naturaleza humana ${ }^{35}$.

Los seres humanos también reconocen las ventajas de tener un animal de compañía con habilidades sociales que le han permitido crear una relación tan amplia que va desde perros de trabajo a mascotas de la familia, ofreciéndose ventajas mutuas ${ }^{35}$. Esta relación es posible porque desde un comienzo el perro perteneció a una especie social, entendiendo por socialización al proceso de aprendizaje por el cual un individuo aprende a aceptar la proximidad de un "conespecifico" (de su propia especie) o la proximidad de otras especies ${ }^{3-11-31-44}$.

Este aprendizaje se produce en un tiempo limitado de la vida del individuo, que se extiende desde la gestación hasta aproximadamente los dos meses de la 
vida del perro (socialización primaria), con un segundo período de socialización durante la pubertad. En este último, el animal reconoce al sexo opuesto y comienza a definir su jerarquía ${ }^{31}$. La vida social brinda beneficios no sólo al individuo sino también a la especie. Permite una mejor distribución de los recursos, tanto de aquellos que son indispensables para la vida: comida, agua, confort térmico, sino también aquéllos que permiten la perpetuación de la especie: el comportamiento sexual y el materno filial. En biología, las motivaciones individuales están recompensadas solo en virtud de la perpetuación de la especie y las situaciones placenteras aseguran el bienestar individual ${ }^{4}$.

Estos recursos son los que la "ciencia del bienestar" propone reconocer y evaluar para determinar cómo se siente ese individuo en un momento determinado. Como seres humanos, los hombres deberíamos cuestionarnos si todos los perros quieren o necesitan las mismas cosas. ¿Es igual un perro salvaje que uno que nació en un departamento? Si la respuesta fuera no, ¿es correcto recoger un perro en la ruta y llevarlo a vivir a un departamento? ¿Está bien llevar a un perro de departamento al campo y allí abandonarlo para que viva libremente? Podemos creer que estrés y bienestar son conceptos antagónicos, sin embargo en el día a día, se suceden situaciones en las cuales las emociones y los comportamientos son cambiantes y donde el bienestar y el estrés son conceptos complementarios ${ }^{59}$.

Deberíamos entender que no todos los perros necesitan las mismas cosas, y que tienen motivaciones diferentes. A continuación analizaremos algunos de estos puntos, intentando considerar las necesidades del mayor número de perros, abarcando este amplio abanico que tiene como punto de análisis la relación humano animal y teniendo en cuenta las necesidades básicas propuestas por la ciencia del bienestar animal. De una manera sesgada se suele confundir bienestar con salud, cuando en realidad el bienestar es mucho más que estar bien físicamente ${ }^{13} \mathrm{y}$ donde la salud, los comportamientos y las emociones se suman en respuesta a su interrelación con el ambiente ${ }^{10}$.

\section{LA COMIDA Y LA NUTRICIÓN}

Si evaluamos el bienestar desde el punto de vista etológico y consideramos los comportamientos naturales de la especie de la cual se origina, surge que el perro es un cazador social, preferentemente de grandes presas. Para obtener su comida, el lobo gris (Canis lupus familiaris) organiza partidas de caza en grupos de varios individuos. Una vez identificada la presa, el líder convoca al resto de la manada mediante movimientos de cola y aumento de su actividad. La estrategia de caza varía según sean grandes presas (rumiantes de porte voluminoso) o pequeñas presas (conejos, gatos, pájaros e incluso peces). En el primer caso, los animales rodean a la presa para evitar su huída y luego la atacan. En el segundo caso, luego de detectarla, la persiguen durante varios kilómetros ${ }^{1}$.
El éxito en la captura de pequeñas presas es muy bajo, entre 25 y $63 \%$, pero es una alternativa para cuando escasean los grandes ungulados. En el caso de las presas voluminosas, lo primero que se devora es el contenido ruminal, conformado por partículas vegetales parcialmente digeridas, luego comen las vísceras y por ultimo, después de varios días, la masa muscular. El animal dominante comerá en primer lugar, luego lo harán los animales de menor escala jerárquica. Llamativamente, los perros domésticos mantienen ese comportamiento considerado como atávico y tratado por los etólogos clínicos como si fuera una patología conductual, la agresión predatoria ${ }^{1}$.

Desde el punto de vista adaptativo, el perro doméstico es considerado como un animal carnívoro optativo, potencialmente omnívoro ("carroñero") en el caso de que las circunstancias lo demanden ${ }^{56}$. No está mal que un perro se alimente de deshechos de la comida humana, pero clínicamente el estado nutricional dependerá de la calidad de la comida basada en la proporción de proteínas, hidratos de carbono y lípidos, así como en su estructura de alimento seco, semihúmedo o húmedo ${ }^{50}$.

En el caso de la alimentación de las mascotas se destacan dos situaciones que pueden afectar la conducta digestiva. Por un lado, en los países desarrollados, la industria relacionada con las mascotas ha dado lugar a la comercialización masiva de los alimentos balanceados. Marcas, formas, colores (según etapas de crecimiento), se venden en veterinarias, supermercados o almacenes de barrio, en pellets sueltos o envasados. De diferentes precios y calidades, algunos de ellos ni siquiera cumplen con los requisitos mínimos que se necesitan para satisfacer el estado nutricional o el control de calidad mínimo ${ }^{23}$.

El consumo de alimentos comercialmente preparados ha aumentado drásticamente en la última década. Aparentemente, el 90\% de las calorías de la alimentación de las mascotas de Estados Unidos, Japón, Australia, Nueva Zelanda y norte de Europa, proviene de los alimentos balanceados. En cambio, en América Latina, la zona del Pacífico y el resto de Europa, es del 30 al $50 \%{ }^{14}$. Al momento de alimentar a las mascotas deberían tenerse en cuenta exclusivamente los alimentos palatables y saludables ${ }^{14,58}$.

\section{ANOREXIA Y MALNUTRICIÓN}

En los perros, el estrés ambiental puede ocasionar una sintomatología compleja que, entre otras reacciones, cursa con hiporexia o anorexia. Tal situación se constata con mayor frecuencia en perros que desarrollan ansiedad por separación o estrés ${ }^{29}$. Sin que los canes dejen de comer, como resultado de una mala calidad de alimentos, se pueden observar situaciones clínicas de desnutrición. En este caso, la malnutrición se define como un desorden de la nutrición que resulta de una inadecuada alimentación o de un desbalance en la ingesta. Esto no solo incluye la caquexia como forma extrema de la pérdida de peso, pues la subnutrición 
puede causar desde alteraciones del manto piloso hasta anemias crónicas. A la inversa, los excesos nutricionales pueden provocar obesidad.

Uno de los problemas que se puede observar en la clínica, relacionado con una malnutrición no intencional provocada por el propietario, tiene que ver con las patologías del desarrollo, comunes en razas gigantes como el gran danés, fila y ovejero alemán, entre otros. En estos casos, el propietario cree que, para crecer más rápido, sanos y con huesos fuertes (ese es el slogan de una propaganda que confunde a los tenedores de mascotas), debe agregar poli vitamínicos y calcio. En realidad, esto es un error que perjudica al desarrollo. En la fase de crecimiento, los huesos deberían ser más blandos para resistir el peso de una masa muscular que está creciendo rápidamente ${ }^{50}$.

Dietas ricas en proteínas sólo generan que la masa muscular crezca más rápido, mientras que el agregado de calcio provoca su precipitación en la hidroxiapatita del hueso y los torna más rígidos. En ambos casos las consecuencias se manifiestan en patologías osteomusculares como la distrofia o los radios curvos ${ }^{50}$. Al considerar los requerimientos, también debe tenerse en cuenta la edad del animal (cachorro, adulto, geronte), así como su actividad (hembra preñada, hembra amamantando, práctica deportiva, animal de compañía u otras).

En oportunidades, cuando el dueño pertenece a un movimiento particular como ser vegetariano, vegano o especialista en dietas, los perros comen comida casera de acuerdo a lo que el dueño dispone. Los vegetarianos hacen comidas balanceadas usando huevos e insumos derivados de la leche, los cuales carecen de algunos aminoácidos que los perros necesitan (arginina, metionina, taurina, triptófano), así como hierro, calcio, zinc, vitamina A y algunas vitaminas del grupo B) ${ }^{19}$ induciendo a un estado de desnutrición documentada clínicamente. Ser vegano o vegetariano es una elección basada en lo cultural. El hombre opta. Sin embargo, cuando se obliga a una mascota a esta opción, tal actitud afecta directamente al estado de salud. Esta circunstancia es un ejemplo de cómo la interpretación del bienestar de un animal queda contextualizada a una cultura ${ }^{41}$.

\section{CAQUEXIA Y OBESIDAD}

Perros emaciados y hambrientos son comunes en muchos países. Estos animales no tienen dueño o bien sus dueños no los alimentan correctamente o son animales salvajes que compiten con las especies silvestres ${ }^{22}$. Cuando el hambre se prolonga durante un tiempo se pueden dar dos situaciones. Una de ellas tiene que ver con la perdida de la masa corporal, acompañada de la disminución o cese de la aptitud reproductiva; la otra se vincula con la aparición de enfermedades infecciosas o parasitarias. Cuando esto sucede, los animales se enferman, sufren o mueren como consecuencia de las complicaciones secundarias. La pérdida de peso tam- bién se puede observar en perros que están sometidos a condiciones ambientales adversas ${ }^{29}$.

En 1965 el británico Roger Brambell describió las "cinco libertades", o derechos de los animales domésticos, como la capacidad de poder fácilmente "levantarse, acostarse, asearse, estirarse y darse vuelta" ${ }^{9}$. Años más tarde fueron adoptados por la World Veterinary Association y luego se crea el Consejo de Bienestar para Animales de Granja, señalando que los animales debían estar libres de hambre y sed, en adecuado estado de salud, exentos de incomodidad, libres de dolor, injurias y enfermedad, capaces de poder expresar su comportamiento normal (espacio suficiente, infraestructura adecuada y compañía de animales de su misma especie), exentos de miedo y distrés ${ }^{10,11}$.

Otro problema nutricional es la obesidad, que surge cuando el balance energético positivo se mantiene durante un tiempo prolongado. Influyen factores como la esterilización, algunas enfermedades metabólicas, los hábitos sedentarios (especialmente falta de ejercicio físico y de acceso al exterior de la vivienda) ${ }^{29}$.

Muchos animales pueden mantener un balance entre ingreso alimentario y actividad física, conservando el peso optimo. No obstante, el peso corporal puede aumentar por factores individuales como el genotipo, el grado e actividad y el tipo de comida. Otros perros, en cambio, no pueden mantener el peso por tener asociada alguna patología conductual como las estereotipias. En este caso, el animal come compulsivamente, no porque hubiera una necesidad fisiológica, sino porque el centro de la saciedad no funciona adecuadamente. La mayoría de estos trastornos obsesivos se relaciona con aburrimiento o falta de ejercicio como consecuencia del encierro. Enriquecer el ambiente con juguetes, asociados o no a la oferta de comida, o aumentar la frecuencia de los paseos, son algunas de las alternativas que los profesionales deberían sugerir para mejor el estado de salud ${ }^{29,51}$

\section{COMIDA Y ENTORNO SOCIAL}

Continuando con aspectos del bienestar en torno a la comida, en los animales sociales adquieren relevancia la disposición del alimento, la forma en que se come y el orden temporal. El perro de mayor jerarquía es el que come en primer lugar, aprovechando la mejor parte de la presa. Lo opuesto ocurre con el animal de jerarquía inferior, que come por último, habitualmente los restos. Con frecuencia, en una actitud antropocéntrica y justificada en el principio errado de que todos somos iguales, los perros comen al mismo tiempo que el propietario. El antropomorfismo es el uso de características humanas para explicar o tratar a los animales no humanos. En los estudios de comportamiento animal hay un consenso casi oficial de que el antropomorfismo debe ser evitado ${ }^{24}$.

Probablemente el momento en que se le da la comida es uno de los rituales más importantes para mantener la jerarquía social, la cual es necesaria para conser- 
var la estabilidad social del grupo de caninos. Quienes así no lo entiendan propiciarán problemas en la salud conductual como agresiones entre perros, ataque a las personas y miedo, cuando no anorexia o bulimia. Los conflictos generados alrededor de la comida son muy frecuentes y se deben a la dificultad de expresar el comportamiento normal (predar, carroñar), fenómenos conflictivos que, cuando se producen, generan disputas entre vecinos.

\section{DE LA REACTIVIDAD O DISTANCIA}

Las especies sociales invierten mucho tiempo permaneciendo en proximidad de otros individuos, particularmente de su misma especie. En el caso de los perros que viven en manadas, del 43 al $85 \%$ del tiempo permanecen a menos de 50 pulgadas uno del otro. Si consideramos que la familia es parte de la manada, entonces el perro tendría que estar más de la mitad de su tiempo en estrecho contacto con el ser humano o con otro perro ${ }^{36}$.

Esto es impracticable para una familia tipo, donde los adultos deben trabajar o invierten su tiempo en actividades varias. Por otra parte, se ha documentado que las patologías conductuales en caninos han aumentado precisamente a partir del momento en que los perros conviven de manera mas estrecha con las personas ${ }^{36,42}$. Lejos de ser una paradoja, esto se debe a que el ser humano interpreta mal las señales de comunicación del perro con quien convive en contacto estrecho; atribuyendo connotaciones humanas a señales propias de los caninos ${ }^{44}$.

Por ejemplo, si un perro macho duerme en la cama con su dueña, es de esperar que no deje que otro macho ocupe un lugar en esa cama a la que considera su territorio. Esto no es por celos, simplemente ocurre porque esta protegiendo su recurso sexual ${ }^{44}$. Otro ejemplo tiene que ver con los paseos. Los perros son animales ritualizados, donde la repetición de las tareas genera estabilidad emocional ${ }^{51}$, sin embargo los propietarios creen que modificar el horario o recorrido del paseo, así como la frecuencia de las caricias, es bueno para no hacer "aburrida la relación". Estas modificaciones pueden generar ansiedad ${ }^{36}$.

\section{TERRITORIO Y ÁREA DE DESCANSO}

El término territorio se refiere a una parte del hogar que es activamente defendida. Ese lugar queda definido por señales olfativas y visuales (orina, marcas de rascado, pozos) ${ }^{39}$, No obstante, los perros pueden vagabundear fuera de su territorio. Estas excursiones pueden ser en cualquier dirección y por distancias de hasta $8 \mathrm{~km}$. Las horas de más actividad son temprano en la mañana (entre las 7 y las 11) y a la tarde (entre las 8 y las 11$)^{46}$.

La conducta de vagabundeo es parte de la actividad de los perros. Si a eso se le suma que son carroñeros, es lógico pensar que van a escarbar y comer de los tachos de basura. Un perro no rompe los tachos de basura porque tiene hambre fisiológica, lo hace porque es parte de su repertorio conductual, lo que genera un conflicto de intereses en torno al recurso territorial y área de descanso, que perjudica la relación entre vecinos ${ }^{56}$.

Cuando se trata del espacio destinado al descanso dentro del hogar, es importante destinar un lugar propio para el perro, que no sea cercano a una puerta utilizada con frecuencia por los propietarios ${ }^{8,31,44}$, preferentemente un sitio elevado y confortable ${ }^{43,51}$, pero que no sea la cama del dueño o los pasillos que van a la habitación de los dueños ${ }^{44}$. Esto facilitará una estabilidad emocional ${ }^{51} \mathrm{y}$ evitará conflictos sociales ${ }^{44}$.

\section{CONTACTO SOCIAL}

Los perros son animales sociales que pasan mucho tiempo con otros perros, otros animales y seres humanos. El contacto social no es una preferencia, es una necesidad. El grado de aproximación a los otros se aprende en el periodo sensible de la vida del individuo ${ }^{27}$. El perro no solo aprende a tolerar a otro, sino que también construye su sistema de comunicación. Todos los mamíferos sociales disponen de los mismos sentidos: tacto, olfato, gusto y vista ${ }^{12}$. No obstante, todos le otorgan una importancia diferente a cada sentido, de acuerdo a las necesidades adaptativas de la especie ${ }^{37}$.

Aprenden a usarlos según lo enseñado por los padres. En el caso particular del perro este aprendizaje se produce durante el apego mutuo (madre/hijo), período que va desde los 15 días de vida (cuando los cachorros comienzan a abrir los ojos y utilizar los oídos) hasta los 60 días. En este momento, el cachorro aprenderá a qué especie pertenece, cómo se comunican los perros y cuáles son los rituales jerárquicos. El adulto modulador le enseñará cuales son las especies amigas y cuáles no ${ }^{44}$.

Obsérvese que en el párrafo anterior se ha mencionadoa un adulto modulador y no a la madre. Sucede que el perro es una especie altricial (aquélla cuyas crías nacen inmaduras, no pueden ver ni oír, tampoco controlar su temperatura corporal), por lo que, hasta que son independientes y pueden comer por sus propios medios, dependerán de cualquier adulto, se apegan a cualquier cosa que les brinde comida, refugio y protección (otros perros, otras especies como el gato o el ser humano).

Debemos aclarar que mediante el apego se adquiere el sistema de comunicación, el cual forma parte del proceso mayor de aprendizaje, que es la socialización. Entender la comunicación de la especie hace que se comporte como perro, pero como perro puede estar socializado con otras especies o con las personas ${ }^{54}$.

La socialización con otros perros se produce principalmente gracias al comportamiento materno-filial. Generalmente, las camadas de perros incluyen de tres a ocho cachorros. Los hermanos conforman un grupo y la madre controla los diferentes comportamientos. Una situación muy interesante es la del comportamiento so- 
cial ingestivo. A partir de los 18 días comienzan a salir los incisivos y los cachorros estarían en condiciones de ingerir comida sólida. En estado natural la madre les llevará pequeñas presas o regurgitará la comida en el nido. Una vez que los cachorros terminan de comer, la mamá les limpiará las comisuras bucales, los pondrá "panza arriba" y estimulará la micción y la defecación.

Mediante este proceso los cachorros aprenden no solo a comer sino también a adoptar la postura de sometimiento ${ }^{53}$. Puede ser que algunos de ellos continúen mamando, aunque con los nuevos dientes aparece el peligro de morder a la madre. Como respuesta a ese dolor agudo, la madre los sanciona con gruñidos y mordiscos. En este proceso los cachorros aprenden a inhibir la mordida ${ }^{11,31,44,53}$.

Los seres humanos no siempre entienden estos rituales y consideran que la hembra es una mala madre porque regurgita la comida sobre los cachorros y "ensucia" la casa, o que tienen que destetarla porque muerde a sus cachorros por "falta de leche". En los primeros dos meses de vida, el cachorro necesita mantenerse con su madre biológica, en un ambiente progresivamente estimulado por la presencia de niños, adultos, juguetes y otras mascotas, evitando la sobre estimulación ${ }^{7}$.

Una situación grave es la que se genera en los criaderos "fábrica de perros", donde se destetan cachorros de menos de 21 días solo por intereses comerciales. En este caso, los perros son mantenidos en cajas o correderas, con un contacto mínimo con los seres humanos o con enriquecimiento ambiental de alguna clase ${ }^{30}$. Numerosos informes sugieren que estos cachorros, criados en total aislamiento, presentan alteraciones de conducta y anormalidades psicológicas persistentes ${ }^{28}$. Debe tenerse en cuenta que tanto el vendedor como el comprador son igualmente culpables de esta situación de crueldad tanto para la madre como para los cachorros, que sufrirán un temprano desapego.

En este momento también se produce la socialización con otras especies y nos interesa, en forma particular, la especie humana. Los perros ven en cada etapa del ser humano a un individuo diferente. Un hombre blanco, amarillo o negro es solo un hombre. En cambio un hombre es diferente a una mujer, a un adolescente, a un niño o a un bebé. Si el perro no entra en contacto con cada etapa del ser humano antes de los dos meses de edad, al llegar a la adultez no será capaz de reconocerlo ${ }^{25}$.

Para el común de la gente esta situación es difícil de comprender, pero es así. Un perro criado en un departamento, aislado de un entorno enriquecido, solo reconocerá a los que viven a su alrededor. Por lo tanto, el Rottweiler que atacó a un bebé solo es culpable de no reconocerlo como especie amiga. El culpable final de la agresión es el propietario que no completó la etapa de socialización. Este perro de socialización incompleta, no será capaz de relacionarse con otros $y$, por haber vivido en confinamiento completo, tampoco será capaz de obtener comida por sí mismo. Esta situación se relaciona con una de las patologías conductuales más complejas y cuyo tratamiento muchas veces es imposible: la agresión a los seres humanos y la agresión a otros perros ${ }^{36}$.

El otro extremo es el de los perros que nacen en un contexto de semi-dependencia ya sea asilvestrado o salvaje. Durante la etapa de socialización no estuvieron en proximidad de un ser humano, por lo tanto, cuando vean a una persona pueden desarrollar miedo $\mathrm{y} / \mathrm{o}$ ansiedad. Cualquiera de estas dos patologías está relacionada también con agresión a los seres humanos y a otras especies ${ }^{40}$. Hombres y mujeres adoptamos una posición antropocéntrica cuando evaluamos la socialización del perro, y más aún cuando la relacionamos con las patologías conductuales ${ }^{42}$.

Los cánidos viven en grupos de 5 a 8 individuos, entre machos y hembras, donde las jerarquías cambian de posición según el recurso (un individuo puede ser dominante a la hora de disponer de la comida, y sumiso ante el recurso territorio o el sexual). Por otra parte, esta posición no es estable a lo largo del año y varía según la disponibilidad de comida, época de celo ${ }^{45} \mathrm{u}$ otras modificaciones ambientales ${ }^{46}$. Cuando los animales viven libres, la conformación de grupos se hace por preferencias, pero cuando viven en un entorno familiar, es la movilidad del grupo humano quien modifica estas relaciones.

Viajes, trabajo, nacimientos, amigos, son factores que en poco tiempo pueden provocar modificaciones del ambiente, sin que el perro entienda el porqué de la ausencia o presencia de sus allegados. Algo semejante ocurre con las perreras, pero allí, las ausencias o presencias sin previo aviso son de otros perros y las jerarquías modifican generalmente mediante fenómenos de agresión. Tal agresión culmina en accidentes con daño físico, cuando no con lesiones irreparables que llevan a la muerte.

Los perros tienen derecho a vivir bien, en un entorno social adecuado a su origen, Un perro asilvestrado o salvaje no debería ser adoptado en un entorno social urbano y, por el contrario, un perro adaptado a vivir en un hábito social limitado, no debería ser liberado a un ambiente natural.

\section{EPÍLOGO}

El trato que los seres humanos prodigan a los animales es una preocupación de la sociedad actual ${ }^{2}$. Los animales importan. Importan por ellos mismos, pero no siempre comprendemos qué es lo que está mal y qué podríamos hacer para evitarlo. Por otra parte, la historia cultural de un pueblo gravita al momento de justificar la forma en que ese grupo social trata a sus animales ${ }^{41,52}$. La ciencia del bienestar evalúa los comportamientos, las emociones y la salud ${ }^{16}$. La filosofía tiene en cuenta y desarrolla teorías éticas que permiten articular el cuidado y la forma en que la comunidad se relaciona con los animales ${ }^{2,20}$. La filosofía y la jurisprudencia se entrelazan en su intento de mejorar la situación actual de millones de individuos, miles de especies, cientos de ecosistemas, un solo mundo. 
La adaptación del perro a la vida social humana se conoce con el nombre de domesticación. Este proceso continuo hace que haya diferentes grados de socialización del perro. Comprender la relación del perro con el ser humano nos permitirá ser menos antropocéntricos $\mathrm{y}$, probablemente, menos crueles ${ }^{17}$.

Nadie puede dudar que los animales poseen un nivel de complejidad individual, social y poblacional, que son concientes de su propia existencia y de la existencia de su especie. Se afirma que los animales deberían incluirse en un círculo moral ampliado, donde nuestra compasión no justifique sino que sea un motivo para considerar lo mejor para los ellos ${ }^{52}$. Pero ¿somos capaces de comprender qué motivaciones les generan placer a los perros? ¿comprendemos realmente qué es lo que desean? ¿la compasión es suficiente para tomar buenas decisiones?

Más allá de los diferentes puntos de vista, los animales deben ser protegidos. Si bien es un gran paso el hecho que se reconozca a los animales como individuos con nombre propio ${ }^{55}$, ello no es suficiente. Se debe educar sobre conceptos básicos acerca de las necesidades de los animales para evitar que sufran innecesariamente, educar sobre el no abandono, sobre el control de la reproducción, sobre la erradicación de las patologías hereditarias. Solo a partir de allí las posiciones éticas serán motivo de discusión y la jurisprudencia podrá obrar de manera adecuada.

\section{REFERENCIAS}

1. Askew HR. 2005. Tratamiento de los problemas de comportamiento en perros domésticos, $2^{\circ}$ ed., Intermédica, Buenos Aires, p. 81-97.

2. Barnard C. 2007. Ethical regulation and animal science: why animal behaviour is special. Anim Behav 74: 5-13.

3. Beaver BV. 1999. Canine Behavior: A guide for veterinarians, $1^{\circ}$ ed., Saunders Co., Philadelphia, p. 43-105.

4. Boissy A. et al. 2007. Assessment of positive emotions in animals to improve their welfare. Physiol \& Behav 92: 375-397.

5. Boitani L, Ciucci P, Ortolani A. 2007. Behaviour and social ecology of free-ranging dogs. In: The behavioural biology of dogs, Ed. Jensen-CABI, London, p. 147-165.

6. Balon EK. 1995. Origin and domestication of the wild carp, Cyprinus carpio: from Roman gourmets to the swimming flowers. Aquacult 129: 3-48.

7. Battaglia C. 2009. Periods of early development and the effects of stimulation and social experiences in the canine. $J$ Vet Behav 4: 203-210.

8. Bowen J, Heath S. 2005. Canine fear, anxiety and phobiarelated disorders. In: Behaviour Problems in Small Animals (Bowen J, Heath S. Ed.), Elsevier, p. 73-95.

9. Brambell Committee. 1965. Report of the technical committee to enquire into the welfare of animals kept under intensive livestock husbandry system. The Brambell report, December 1965, London.
10. Broom DM. 1999. Animal welfare: the concept \& the issues. In: Attitudes to Animals: Views in Animal Welfare (Dolins F.L. ed.), University Press, Cambridge, p. 129-142.

11. Coll V. 2005. Evolución psíquica, educación y patologías del comportamiento. En: Neonatología y pediatría canina y felina (Prats, A. ed.), Inter-Médica, Buenos Aires, p. 451475.

12. Coppinger RP, Coppinger L. 2001. Dogs: a new understanding of canine origin, behavior and evolution, University of Chicago Press, Chicago, USA.

13. Christiansen SB, Forkman B. 2007. Assessment of animal welfare in a veterinary context. A call for ethologist. Appl Anim Behav Sci 106: 203-220.

14. Crane SW, Griffin RW, Messent PR. 2000. Introducción a los alimentos comerciales para mascotas. En: Nutrición clínica en pequeños animales, $4^{\circ} \mathrm{Ed}$, Panamericana, Colombia, p.128-147.

15. Daniels TJ, Bekoff M. 1989. Feralization: the making of wild domestic animals. Behav Processes 19: 79-84.

16. Dawkins MS. 2006. Through animal eyes: what behaviour tell us. Appl Anim Behav Sci 100: 4-10.

17. Dotson MJ, Hyatt EM. 2008. Understanding dog-human companionship. J Busin Res 61: 457-466.

18. Downs JF. 1960. Domestication: an examination of the changing social relationships between man and animals. In: Animal Domestication \& Behavior, CAB Internat., p. 21.

19. Food and Drug Administration Center for Veterinary Medicine. 2002. Report of February 28. On line: www. fda.gov/cvm/FOI/DFreport.

20. Fraser D. 1999. Animal ethics and animal welfare science: bridging the two cultures. Appl Anim Behav Sci 65: 171189.

21. Galibert F, Quignon P, Hitte C, André C. 2011. Toward understanding dog evolutionary and domestication history. Compts Rendus Biol 334: 190-196.

22. Galo ZR, Branch LC. 2016. Altered activity patterns and reduced abundance of native mammals in sites with feral dogs in the high Andes. Biol Conserv 193: 9-16.

23. Gazzotti T, Biagi G, Pagliuca G, Scardilli PM, Grandi M, Zaghini G. 2015. Occurrence of mycotoxins in extruded commercial dog food. Anim Feed Sci \& Technol 202: 81-89.

24. Horowitz AC, Bekoff M. 2007. Naturalizing anthropomorphism: Behavioral prompts to our humanizing of animals. Anthrozoos 20: 23-35.

25. Howell TJ, Bennett PC. 2011. Puppy power! Using social cognition research tasks to improve socialization practices for domestic dogs (Canis familiaris). J Vet Behav 6: 195204.

26. Jenesen P. 2007. El perro en su nicho: entre humanos. In: The Behavioural Biology of Dog, Ed.,CABI, London, p. 208.

27. King, T, Marston LC, Bennett PC. 2012. Breeding dogs for beauty and behaviour: Why scientists need to do more to develop valid and reliable behaviour assessments for dogs kept as companions. Appl Anim Behav Sci 137: 1-12. 
28. Latham NR, Mason GJ. 2008. Maternal deprivation and the development of stereotypic behaviour. Appl Anim Behav Sci 110: 84-108.

29. Mariotti VM, Amat M, Herver M, Baucells M, Manteca X. 2009. Factores ambientales implicados en el control de la conducta del perro y del gato: alimentación, manejo y ejercicio. Clin Vet Peq Anim 29: 209-215.

30. McMillan FD, Duffy DL, Serpell JA. 2011. Mental health of dogs formerly used as breeding stock in commercial breeding establishments. Appl Anim Behav Sci 135: 86-94.

31. Manteca Vilanova, X. 2003. Comportamiento normal del perro. In: Etología Clínica Veterinaria del perro y gato, ed. Multimédica, $3^{\text {a }}$ Ed., Barcelona, p. 9-87.

32. Matter HC, Daniels T. 2002. Dog ecology and population biology. In: Dogs, Zoonoses and Public Health, Ed. MacPherson, UK, p. 17-62.

33. Mehrkam LR, Wynne CD. 2014. Behavioral differences among breeds of domestic dogs (Canis lupus familiaris): Current status of the science. Appl Anim Behav Sci 155: 12-27.

34. Miklósi Á. 2007. Dog Behaviour, Evolution and Cognition, Oxford University Press, USA, 274 p.

35. Miklósi A, Topal J. 2013. What does it take to become 'best friends'? Evolutionary changes in canine social competence. Trends Cognit Sci 17: 287-294.

36. Mills D, Zulch H. 2010. El papel del miedo y de la ansiedad en el comportamiento agresivo de los perros. Vet Focus 20: 44-49.

37. Mogi K, Nagasawa M, Kikusui T. 2011. Developmental consequences and biological significance of mother-infant bonding. Progr Neuro Psych Pharm 35: 1232-1241.

38. Morey DF. 2006. Burying key evidence: the social bond between dogs and people. J Archaeol Sci 33: 158-175.

39. Morgan KN, Tromborg CT. 2007. Sources of stress in captivity. Appl Anim Behav Sci 102: 262-302.

40. Muller G. 2000. Los trastornos de comportamiento durante la crianza en el perro. Le Point Vétérin 31: 109-116.

41. Ohl F, Van der Staay FJ. 2012. Animal welfare: at the interface between science and society. The Vet J 192: 13-19.

42. Overall KL, Dyer D. 2005. Enrichment strategies for laboratory animals from the viewpoint of clinical veterinary behavioral medicine. ILAR Journal 46: 202-216.

43. Overal KL. 2011. The dog is smarter than you know: advances in understanding canine learning, memory and cognition. Doi: 10.1053/j.tcam.2011.01.001.

44. Pageat P. 2000. Patología del comportamiento del perro, Pulso Ediciones SA, $2^{\circ}$ Ed., Barcelona, p: 9-42.

45. Pal SK, Ghosh B, Roy S. 1998. Agonistic behaviour of free-ranging dogs (Canis familiaris) in relation to season, sex and age. Applied Anim Behav Sci 59: 131-148.

46. Pal SK, Ghosh B, Roy S. 1999. Inter- and intra-sexual behaviour of free-ranging dogs (Canis familiaris). Appl Anim Behav Sci 62: 267-278.
47. Parker HG. 2004. Genetic structure of the purebred domestic dog. Science 304: 1160-1190.

48. Price EO. 1999. Behavioral development in animals undergoing domestication. Appl Anim Behav Sci 65: 245-271.

49. Price EO. 2002. Adaptation to the biological environment. En: Anim Domest \& Behav, CABI publishing, UK, p. 95130.

50. Richardson DC, Zentek J, Hazewinkel HA, Toll PW, Zicker SC. 2000. Enfermedad ortopédica del desarrollo en perros. In: Nutrición clínica en pequeños animales, $4^{\circ}$ Ed, Panamericana, Colombia, p.595-622.

51. Rooney N, Gaines S, Hiby E. 2009. A practitioner's guide to working dog welfare. J Vet Behav 4: 127-134.

52. Ryder RD. 1992. Painism: the ethics of animal rights and the environment In: Welfare and the environment, RSPA, Gran Bretaña, p. 196-210.

53. Seksel K, Mazurski EJ, Taylor A. 1998. Puppy socialization programs: short and long term behavioural effects. Appl Anim Behav Sci 62: 335-349.

54. Seksel K. 2010. Puppy Socialization. Vet Focus 20: 7-12.

55. Singer P. 2016. En el nombre de todos los animales. Diario Clarin 14-02-16, p. 28.

56. Stafford K. 2007. The welfare of dogs, Springer, Holanda, p. 6-11.

57. Stager LE. 1991. Why were hundreds of dogs buried at Ashkelon? Biblical Archaeology Review, May/June 1992, p. 26-42.

58. Travain T, Colombo ES, Grandi LC, Heinzl E, Pelosi A, Prato E, Valsecchi P. 2016. How good is this food? A study on dogs' emotional responses to a potentially pleasant event using infrared thermography. Physiol \& Behav 159: 80-87.

59. Veissier I, Boissy A. 2006. Stress and welfare: two complementary concepts that are intrinsically related to the animal's point of view. Physiol \& Behav 42: 429-433.

60. Vigne JD. 2011. The origins of animal domestication and husbandry: a major change in the history of humanity and the biosphere. Compt Rendus Biol 334: 171-181.

61. Vila C et al. 1997. Multiple and ancient origins of the domestic dog. Science 276: 1687-1689.

62. Wapnish P, Hesse B. 1993. Pampered pooches or plain pariahs? The Ashkelon dog burials. Biblical Archaeologist 56: $55-80$.

63. Wechsler B, Lea SE. 2007. Adaptation by learning: Its significance for farm animal husbandry. Appl Anim Behav Sci 108: 197-214.

64. World Health Organization. 1988. Report of WHO consultation on dog ecology studies related to rabies control. Ref. WHO/Rab. Res/88.25, Geneva.

65. Zeder MA, Emshwiller E, Smith BD, Bradley DG. 2006. Documenting domestication: the intersection of genetics and archaeology. Trends in Genetics. Doi: 22.10.1016/j.tig. 2006.01.007. 